\title{
Response to Letter: "Do systemic antibiotics for skin and soft tissue abscesses after incision and drainage improve cure rates compared with placebo? - A critical appraisal"
}

Dear Editor,

We are pleased that the CFEM editors highlighted newly published evidence regarding skin and soft tissue abscess treatment in the January 2020, CFEM Journal Club. Ahlin et al. critically appraised a systematic review and meta-analysis by Gottlieb et al., suggesting increased rates of a clinical cure using systemic antibiotics following incision and drainage of skin and soft tissue abscesses. ${ }^{1}$ While the authors provide sound critical appraisal of the meta-analysis, they conclude with a recommendation to add systemic antibiotic therapy with methicillin-resistant Staphylococcus aureus (MRSA) coverage following incision and drainage of abscesses in the emergency department (ED).

We would like to offer a counterargument to this conclusion. The results of this meta-analysis are driven primarily by two important multicentre trials conducted in the United States where the communityassociated MRSA prevalence was $49.4 \%$ and $45.3 \%$, respectively. ${ }^{2,3}$ In Canada, previous work from Borgundvaag et al. underlined important variations in the rate of MRSA, where more than half of the 19 study sites had a prevalence of MRSA $\leq$ $30 \% .{ }^{4}$ Regionally, the CHU de Québec-Université Laval Antimicrobial Stewardship and Resistance Committee reports a $10 \%$ rate of MRSA. Ultimately, MRSA prevalence for purulent skin and soft tissue infections in Canada is much lower in comparison to the United States. It is still unclear whether the addition of systemic antibiotics following abscess incision and drainage results in an increased rate of clinical cure in populations with lower MRSA prevalence. Moreover, the included studies in the meta-analysis were not powered to detect rare but serious adverse events associated with antibiotics nor the potential impact on the emergence of resistant pathogens. Recently, the Canadian Association of Emergency Physicians' Choosing Wisely Canada working group reviewed those trials and still maintained their recommendation to withhold antibiotic treatment following incision and drainage of simple abscesses unless extensive cellulitis exists. $^{5}$

This issue highlights the importance of local antimicrobial stewardship programs and the need for continued research initiatives aiming to improve the care of patients with skin and soft tissue infections. For the moment, we believe more data are needed to support a definitive recommendation for the addition of antibiotics effective against MRSA following incision and drainage of simple abscesses in most Canadian EDs, where the local prevalence of MRSA remains low. Emergency physicians should consider patient factors (e.g., MRSA risk factors, immunocompromise), infection characteristics (e.g., significant surrounding cellulitis), and local prevalence rates when engaging in shared decision-making on the appropriateness of antimicrobial therapy in addition to incision and drainage for skin abscesses.

Pierre-Gilles Blanchard, MD, MSc, PhD, MBA

Faculté de médecine, Département de médecine familiale et de médecine d'urgence, Université Laval, Québec

Krishan Yadav, MD, MSc

Department of Emergency Medicine, University of Ottawa

Ottawa Hospital Research Institute, Ottawa, Ontario 


\section{Geneviève Soucy, MD}

Faculté de médecine, Département de microbiologie-infectiologie et d'immunologie, Université Laval, Québec

Marcel Émond, MD, MSc

Faculté de médecine, Département de médecine familiale et de médecine d'urgence, Université Laval, Québec

\section{Eric Mercier, MD, MSc}

Faculté de médecine, Département de médecine familiale et de médecine d'urgence, Université Laval, Québec
Keywords: Abscess, antibiotic stewardship, skin and soft tissue infections

\section{REFERENCES}

1. Gottlieb M, DeMott JM, Hallock M, Peksa GD. Systemic antibiotics for the treatment of skin and soft tissue abscesses: a systematic review and meta-analysis. Ann Emerg Med 2019;73(1):8-16.

2. Daum RS, Miller LG, Immergluck L, Fritz S, Creech CB, Young D, et al. A placebo-controlled trial of antibiotics for smaller skin abscesses. Massachusetts Medical Society. $N$ Engl $7 \mathrm{Med}$ 2017;376(26):2545-55.

3. Talan DA, Mower WR, Krishnadasan A, Abrahamian FM, Lovecchio F,
Karras DJ, et al. Trimethoprimsulfamethoxazole versus placebo for uncomplicated skin abscess. Massachusetts Medical Society. $N$ Engl 7 Med 2016;374(9):823-32.

4. Borgundvaag B, Ng W, Rowe B, Katz K; EMERGency Department Emerging Infectious Disease Surveillance NeTwork (EMERGENT) Working Group. Prevalence of methicillinresistant Staphylococcus aureus in skin and soft tissue infections in patients presenting to Canadian emergency departments. CFEM 2013;15 (3):141-60.

5. Cheng AHY, Campbell S, Chartier LB, Dowling S, Goddard T, Gosselin $\mathrm{S}$, et al. Choosing Wisely Canada's emergency medicine recommendations: time for a revision. Cambridge University Press. C7EM 2019;21 (6):717-20. 